\title{
Cardiac sarcoma: A Case Report
}

\author{
Tugce Uzar ${ }^{1}$, Kursat Erkaya ${ }^{1}$, Utku Arslan1, Ali E Atas ${ }^{2}$, Pankina Ekaterina ${ }^{3}$, Adem Dirican ${ }^{4}$ and Sevket Ozkaya $^{5 *}$ \\ ${ }^{1}$ Bahcesehir University, Faculty of Medicine, Istanbul, Turkey \\ ${ }^{2}$ Department of Cardiology, Samsun Medical park Hospital, Samsun, Turkey \\ ${ }^{3}$ Department of Pulmonary Medicine, Bashkir State Medical University, Ufa, Russia \\ ${ }^{4}$ Department of Pulmonary Medicine, Samsun Medical park Hospital, Samsun, Turkey \\ ${ }^{5}$ Department of Pulmonary Medicine, Bahcesehir University, Istanbul, Turkey
}

\begin{abstract}
Primary cardiac sarcomas are rare clinic entity. It should be kept in mind in young people with dyspnea because it is a difficult disease to detect radiologically. We aimed to present a 40 years-old female patient with primary cardiac sarcoma. Although the primary cardiac sarcoma is rare, it should be considered in young patients with bilateral pleural effusion.
\end{abstract}

\section{Introduction}

Primary tumours of the heart are rare. About $75 \%$ of primary cardiac tumours are benign mesenchymal tumours, most of which are myxomas. Primary cardiac sarcomas are rare and with an incidence of 0.0017 to $0.019 \%$. Symptoms depend on the chambers and the cardiac structures involved $[1,2]$. We aimed to present a case with cardiac sarcoma. The written informed consents to publish/present these cases were obtained from the patients.

\section{Case Presentation}

A 40-year-old lady, non-smoker, presented with five months history of exertional dyspnoea. Bilaterally pleural fluids were noted on chest radiography and thoracic CT scans (Figure 1). Echocardiography was noted as normal at first evaluation and diagnosed as these symptoms caused due to depression.

After 5 months of first admission, chest roentgenography showed the bilateral reticular opacities (Figure 2) and HRCT showed the multiple pulmonary nodules (Figure 3 ). We suspected from malignancy and F-18 FDG PET/CT scan performed to identify primary tumour and there was malignant uptake in left iliac bone uptake (Figure 4A and 4B). We perform the thoracic MR to evaluate the mediastinal structures and there is a large mass lesion in the right atrium (Figure 5). Intracardiac mass lesion was confirmed at 3rd echocardiography (Figure 6). Cardiac sarcoma was defined with histopathologic examination of iliac bone biopsy.

\section{Discussion}

Primary cardiac sarcomas are malignant neoplasms deriving from mesenchymal cells and confined to the heart at the time of diagnosis [3]. Primary cardiac sarcomas are aggressive tumours that generally do not produce symptoms until they are locally advanced. These delayed manifestations are nonspecific and include dyspnoea, chest pain, congestive heart failure secondary to obstruction of blood flow as well as systemic symptoms [1]. Because of non-specificity of symptoms and rarity of these tumours they are often difficult to diagnose preoperatively

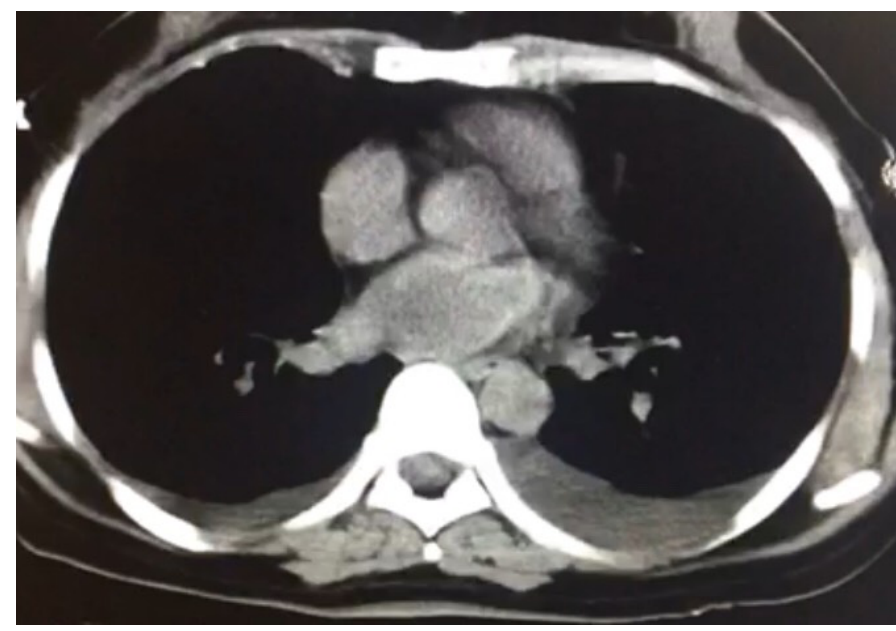

Figure 1. Thoracic CT scan showing the bilaterally pleural effusion. Also, the intra atrial mass lesion was retrospectively noted, after the cardiac sarcoma diagnosis.

and are missed occasionally. The advent of modern investigative tools including transoesophageal echocardiogram, CT scan and cardiac MRI increases the likelihood of preoperative diagnosis [2]. Echocardiography by transthoracic or preferably transoesophageal route is a convenient, rapid and inexpensive tool to identify intracardiac masses [4].

The CT and MRI scans of chest and abdomen are complementary to echocardiography. They have the advantage of showing the extracardiac extent of tumour and presence of metastases in addition to the anatomical details of the lesion. CT scan shows myocardial

${ }^{\star}$ Correspondence to: Sevket Ozkaya, Department of Pulmonary Medicine, Faculty of Medicine, Bahcesehir University, Istanbul, Turkey, Tel: +90-532-4741309; E-mail: ozkayasevket@yahoo.com

Key words: primary cardiac sarcomas, radiology, F-18 FDG PET/CT

Received: January 29, 2019; Accepted: February 18, 2019; Published: February 22,2019 


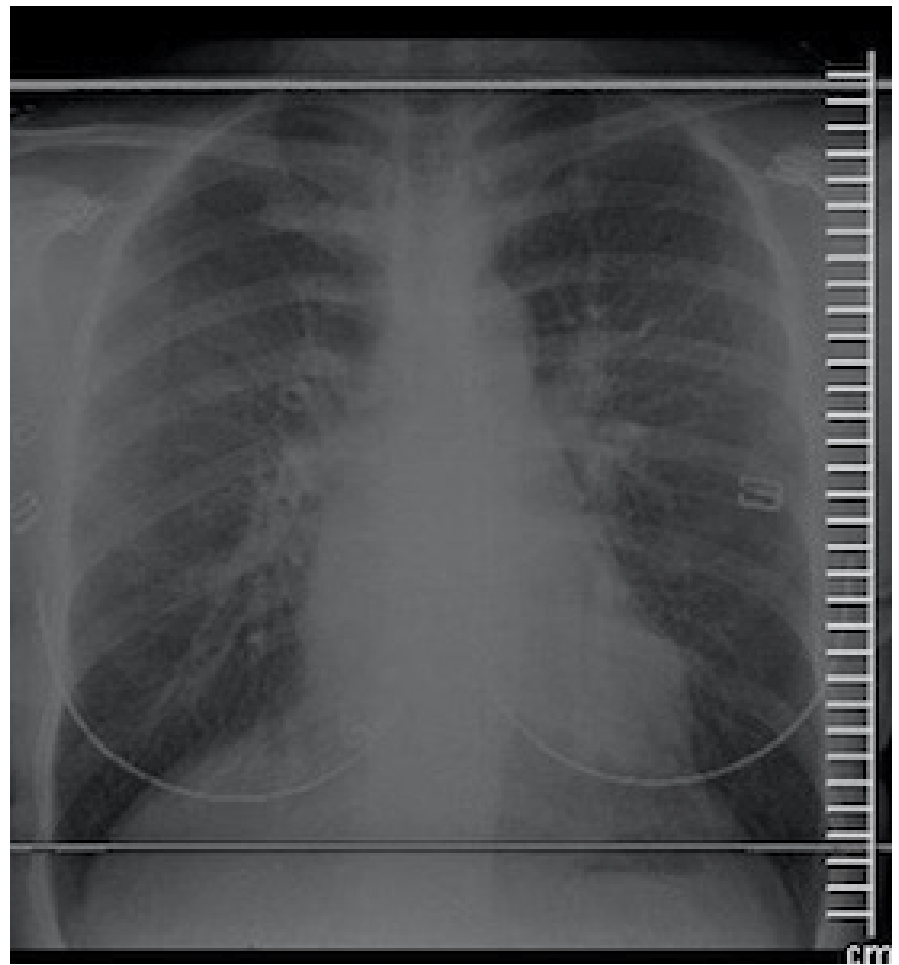

Figure 2. Chest roentgenography showing the bilaterally reticular opacities

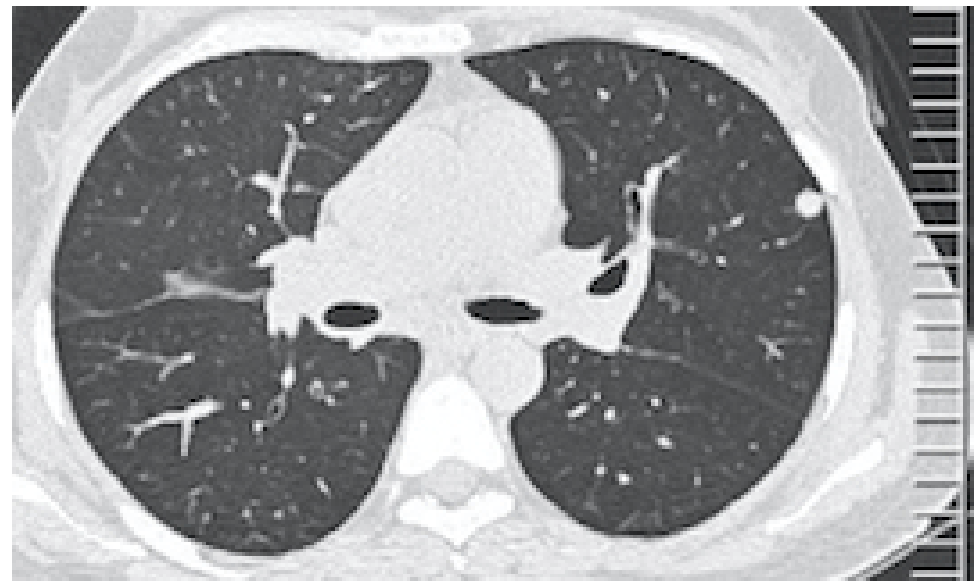

Figure 3. HRCT image showing the solitary pulmonary nodules

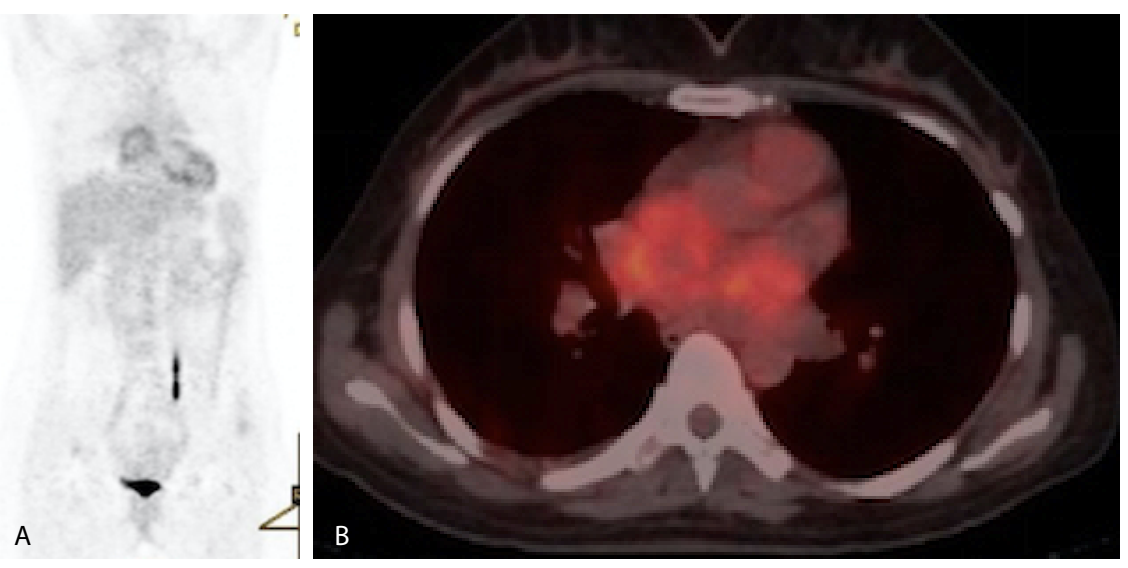

Figure 4A and 4B. F-18 FDG PET/CT scan missed lesion due to physiological cardiac uptake in the lesion 


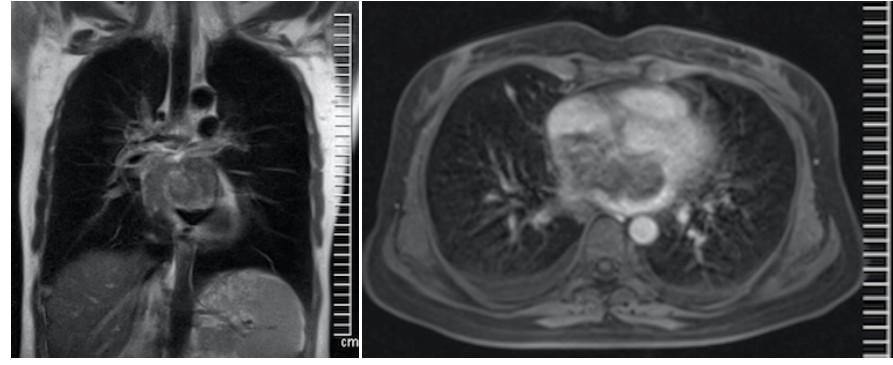

Figure 5A and 5B. Contrast-enhanced Thoracic MR images showing the intracardiac mass

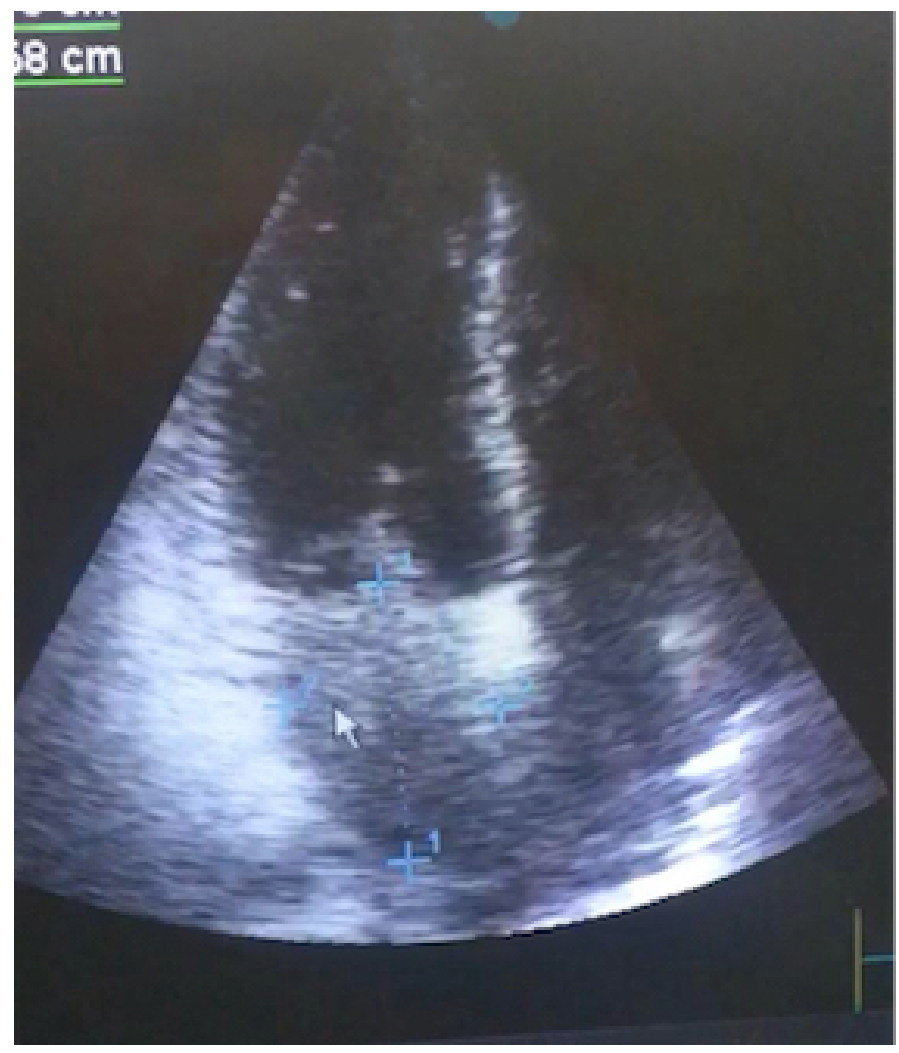

Figure 6. Transthoracic echocardiography image showing the intraartrial mass

infiltration, compression of cardiac chambers along with pericardial and great vessel involvement. MRI is useful to assess tumour volume, tumour burden, mediastinal invasion and response to therapy [2].

Exertional dyspnoea was main symptom in our case. First radiologic finding was bilaterally pleural effusion. Thoracic CT scans and echocardiographic examinations were failed to identification of intracardiac lesion. F-18 FDG PET/CT scan missed lesion due to physiological cardiac uptake in the lesion. Contrast-enhanced Thoracic MR images showed the intracardiac mass and it was confirmed with re-performed echocardiography carefully. And, time of diagnosis of cardiac sarcoma was 5 months after the first admission of clinic.

Wide surgical excision remains the only proven therapy which improves the symptoms and offers the potential for long term survival in selected patients. Role of adjuvant treatment remains undefined. Elective therapy includes complete surgical excision when possible, followed by radio- and chemotherapy. Despite these therapeutic strategies, prognosis of cardiac sarcomas is very poor, with median survival of less than 1 year [5]. Systemic therapies play a minor role in these patients, because their efficacy is limited, and symptoms necessitate immediate surgical action. However, in patients with metastatic disease, systemic treatment should follow the recommendations and guidelines based on the histological diagnosis [6]. Systemic chemotherapy was started in our case due to metastatic sarcoma.

\section{Conclusion}

Although the primary cardiac sarcoma is rare, it should be considered in young patients with bilateral pleural effusion. It should be kept in mind that it is a difficult disease to detect radiologically and echocardiographically.

\section{Ethical approval}

Ethical approval is not required at our institution to publish an anonymous case report.

\section{Consent}

Written informed consents were obtained from the patients for publication of this manuscript and any accompanying images.

\section{Competing interest}

The authors declare that they have no competing interests.

\section{References}

1. Hamidi M, Moody JS, Weigel TL, Kozak KR (2010) Primary Cardiac Sarcoma. Ann Thorac Surg 90: 176-181. [Crossref]

2. Devbhandari MP, Meraj S, Jones MT, Kadir I, Bridgewater B (2007) Primary cardiac sarcoma: reports of two cases and a review of current literature . $J$ of Cardiothorac Surg 24: 34. [Crossref]

3. Orlandi A, Ferlosio A, Roselli M, Chiariello L, Spagnoli LG (2010) Cardiac Sarcomas An Update.

4. J Thorac Oncol 5: 1483-1489. [Crossref]

5. Kim JT, Baek WK, Kim KH, Yoon YH, Kim DH, et al. (2003) A primary cardiac sarcoma preoperatively presented as a benign left atrial myxoma. Yonsei Med J 44: 530-533. [Crossref]

6. Grebenc ML, Rosado de Christenson ML, Burke AP, Green CE, Galvin JR (2000) Primary cardiac and pericardial neoplasms: radiologic-pathologic correlation. Radiographics 20: 1073-1103. [Crossref]

7. Niclauss L, Montemurro M, Kirsch M, Pretre R (2018) Sarcoma of the heart: surviva after surgery. Interact CardioVasc and Thorac Surg 27: 198-201. [Crossref]

Copyright: (C2019 Uzar T. This is an open-access article distributed under the terms of the Creative Commons Attribution License, which permits unrestricted use, distribution, and reproduction in any medium, provided the original author and source are credited. 